J. Dairy Sci. 96:4529-4534

http://dx.doi.org/10.3168/jds.2013-6603

(C) American Dairy Science Association ${ }^{\circledR}, 2013$.

\title{
Technical note: Identification of suitable normalizers for microRNA expression analysis in milk somatic cells of the yak (Bos grunniens)
}

\author{
W. L. Bai, ${ }^{*}$ R. H. Yin, ${ }^{* 1}$ R. J. Yang,† W. A. Khan,‡ Z. J. Ma,§ S. J. Zhao,\# W. Q. Jiang, ${ }^{*} Z$. Y. Wang, ${ }^{*}$ Y. B. Zhu, \\ G. B. Luo, ${ }^{*}$ and Z. H. Zhaot ${ }^{1}$ \\ ${ }^{*}$ College of Animal Science and Veterinary Medicine, Shenyang Agricultural University, Shenyang 110866, China \\ †College of Animal Science, Jilin University, Changchun 130062, China \\ ‡Department of Animal Science, Cornell University, Ithaca, NY 14853 \\ $\S$ Academy of Animal Science and Veterinary Medicine, Qinghai University, Xining 810016, China \\ \#Institute of Biotechnology, Animal Science Research Academy of Sichuan Province, Chengdu 610066, China
}

ABSTRACT

MicroRNA are approximately 18- to 22-nucleotide nonprotein coding molecules that play important roles in the regulation of gene expression at the posttranscriptional level. In the present study, we assessed the suitability of 8 noncoding small RNA as normalizers for microRNA (miR) quantitative analysis in milk somatic cells of lactating yaks, including 3 small nuclear RNA (snRNA; RNU1A, RNU5A, and RNU6B), 3 small nucleolar RNA (snoRNA; SNORA73A, Z30, and SNORA74A), 1 rRNA (5S), and 1 transfer RNA (Met-tRNA). The snRNA RNU1A, RNU5A, and SNORA73A were identified as the most stable references in milk somatic cells of lactating yaks. Also, a minimum of 3 reference RNA ( $R N U 1 A, R N U 5 A$, and SNORA 73A) were required for the normalization of microRNA expression data in milk somatic cells of the lactating yak. We further evaluated the suitability of the combination of RNU1A, RNU5A, and SNORA73A as reference RNA in milk somatic cells of lactating yaks via detecting the relative expression of $\mathrm{miR} 16 \mathrm{~b}$, miR 21-5p, miR 145, and miR 155 as microRNA of putative interest. In comparison to the colostrum period, on the whole, the expressions of the 4 microRNA were found to be upregulated at an early period and, thereafter, a declining pattern was exhibited from early to final periods in all microRNA investigated. Based on the results from this study, we recommend that the combination of RNU1A, RNU5A, and SNORA73A can be used as normalizers for microRNA quantitative analysis in future longitudinal studies on milk somatic cells of lactating yaks in relation to lactation.

Key words: yak, reference RNA, microRNA, milk somatic cells

Received January 21, 2013.

Accepted April 12, 2013.

${ }^{1}$ Corresponding authors: yinronghuan@163.com and zhzhao@jlu. edu.cn

\section{Technical Note}

The yak (Bos grunniens) is considered to be one of the most remarkable domesticated farm animals (Bai et al., 2012). It is distributed in regions of extreme harshness characterized by high altitudes and severely cold winters with no absolutely frost-free periods. Yak milk plays an important dietary role for the local people, and it also forms a major source of household income for residents on the Qinghai-Tibetan Plateau (Liu et al., 2011). In the last few years, yak milk has received increased attention from the ever-growing number of people due to its good quality with higher percentages of protein, organic calcium, and conjugated linoleic acid compared with those of cow milk (Bai et al., 2012).

Micro-ribonucleic acids are endogenous small noncoding RNA molecules of approximately 18 to 22 nucleotides in length. They negatively regulate gene expression mainly through binding to the $3^{\prime}$-untranslated region of target coding mRNA (Du and Zamore, 2005). In recent years, numerous microRNA have also been identified in the mammary glands of different mammalian species, such as mice (Avril-Sassen et al., 2009), cattle (Li et al., 2012), and goats (Ji et al., 2012). For further insight into their potential functional roles, it is essential to elucidate their expression characteristics in the mammary glands of dairy animals. The aim of the present work was to identify suitable references from 8 candidate small noncoding RNA species for studying microRNA expression in milk somatic cells of lactating yaks during different stages of lactation.

Milk samples were collected from 6 adult yaks belonging to the Maiwa breed at d 3 (colostrum period), 28 (early period), 80 (peak period), 150 (late period), and 180 (final period) after calving (Bai et al., 2012). The animals sampled for milk were in good general health, and were free of clinical mastitis. We isolated the somatic cells from raw milk samples according to the assay described by Boutinaud et al. (2002). The SCC of each sample was adjusted to $6 \times 10^{8}$ cells, and 
was stored in Sample Protector (TaKaRa Biotechnology Co. Ltd., Dalian, China) at $-20^{\circ} \mathrm{C}$ until RNA isolation. Total RNA was extracted from the isolated somatic cells using TRIzol reagent (Sangon Biological Engineering Technology \& Services Co. Ltd., Shanghai, China), according to the manufacturer's instructions. To verify RNA integrity, extracts were subjected to $1.5 \%$ agarose electrophoresis. The assessment of quantity and purity of total RNA was carried out with a UV spectrometer at 260- and 280-nm wavelengths, and the optical density (OD) at $260 \mathrm{~nm}: \mathrm{OD}$ at $280 \mathrm{~nm}\left(\mathrm{OD}_{260}: \mathrm{OD}_{280}\right)$ ratio was found to be in the range from 1.8 to 2.0 for all the samples. The RNA $(1 \mu \mathrm{g})$ were first tailed by using poly(A) polymerase, and then the first strand of cDNA was synthesized by using oligo deoxythymine [oligo(dT)] using the One Step PrimeScript microRNA (miRNA) cDNA synthesis kit (no. D350; TaKaRa Biotechnology Co. Ltd.) according to the manufacturer's instructions.

A total of 8 candidate references were chosen, including 3 small nuclear RNA (snRNA; RNU1A, RNU5A, and $R N U 6 B$ ), 3 small nucleolar RNA (snoRNA; SNORA 73A, Z30, and SNORA74A), 1 rRNA (5S), and 1 transfer RNA (tRNA; Met-tRNA). The 4 microRNA (miR) miR 16b, miR 21-5p, miR 145, and miR 155, whose expression patterns were characterized in the mammary gland of cattle (Wang et al., 2012), were also used as interest microRNA to validate the identified optimal references for microRNA expression. The information for primers used was listed in Table 1.

Real-time PCR was performed with the SYBR Premix Ex Taq II kit (TaKaRa Biotechnology Co. Ltd.) on a LightCycler 480 real-time PCR system (Roche Diagnostics GmbH, Mannheim, Germany). A 6-point standard curve was produced for each gene by the 10fold serial dilutions of obtained cDNA. Reactions were carried out in a final volume of $20 \mu \mathrm{L}$, consisting of 10.0 $\mu \mathrm{L}$ SYBR Premix Ex Taq II $(2 \times), 0.8 \mu \mathrm{L}$ of forward primer $(10 \mu M), 0.8 \mu \mathrm{L}$ of reverse primer $(10 \mu M), 2.0$ $\mu \mathrm{L}$ of first-strand cDNA, and $6.4 \mu \mathrm{L}$ of PCR-grade water. The cycling program was as follows: initial template denaturation at $95^{\circ} \mathrm{C}$ for $30 \mathrm{~s}$, followed by 40 cycles of denaturation at $95^{\circ} \mathrm{C}$ for $5 \mathrm{~s}, 53$ to $60^{\circ} \mathrm{C}$ (Table 1) for $20 \mathrm{~s}$, and $72^{\circ} \mathrm{C}$ for $10 \mathrm{~s}$. The last cycle was followed by a melting curve analysis ranging from 65 to $95^{\circ} \mathrm{C}$ as the set default value of instrument to confirm the presence of one gene-specific single peak for each primer set. Each reaction was performed in triplicate, and a negative control without cDNA template was contained in each measurement. The real-time PCR efficiency $(E)$ was calculated using the equation $E=10^{(-1 / \text { slope })}$.

The stability of the 8 candidate references was analyzed by the geNorm (Vandesompele et al., 2002) and NormFinder (Andersen et al., 2004) computational programs. The obtained crossing point $(\mathbf{C p})$ values from real-time PCR were converted following the instructions of the software. The correlation coefficient (r) between the geNorm M-value and the NormFinder stability value was evaluated using SPSS software (SPSS Inc., Chicago, IL). Additionally, the optimal number of references was also determined using the geNorm program (Vandesompele et al., 2002).

Based on the combination of RNU1A, RNU5A, and SNORA 73A against RNU1A alone as references, we detected the expression pattern of miR $16 \mathrm{~b}$, miR 21-5p, miR 145, and miR 155 as microRNA of interest in somatic cells of lactating yaks in the colostrum period, early period, peak period, late period, and final period after calving(Bai et al., 2012). The reaction solution of real-time PCR was prepared in a final volume of $20 \mu \mathrm{L}$ as described above, with an annealing temperature of 61 or $62^{\circ} \mathrm{C}$ (Table 1 ). Relative quantifications of the 4 microRNA were carried out with a geometric mean of $E_{\text {ref }}{ }^{\Delta C P_{\text {ref }}}$ for 3 references (RNU1A, RNORA73A, and $R N U 5 A)$ and $R N U 1 A$ alone as references, respectively, and the relative expression level of the 4 interest microRNA was calculated using the following equation proposed in a previous study by Pfaffl (2001):

$$
\text { Ratio }=\frac{\left(E_{m i r}\right)^{\Delta C P_{m i r}(\text { control-sample })}}{\left(E_{r e f}\right)^{\Delta C P_{r e f}(\text { control-sample })}},
$$

where $E_{\text {mir }}=$ real-time PCR efficiency of interest microRNA, $E_{r e f}==$ real-time PCR efficiency of the reference, $\Delta C P_{\text {mir }}=$ the $\mathrm{Cp}$ difference of control - sample of interest microRNA, and $\Delta C P_{\text {ref }}=$ the Cp difference of control - sample of the reference. Based on comparison with the reference, the ratio of interest microRNA was expressed through a sample (early, peak, late, and final periods) against a control (colostrum period).

Based on the geNorm algorithm, RNU1A and SNORA 73A were identified as the most stable pairwise, and $R N U 5 A$ was ranked as the third most stable reference, whereas $5 S$ and Met-tRNA had the least expression stability in somatic cells of lactating yaks (Figure 1a). The results of NormFinder analysis are shown in Figure 1b. Compared with the results obtained from the geNorm procedure, some differences existed in the ranking of the candidate references. On the whole, however, the ranking was consistent with that of geNorm analysis. The snRNA RNU1A, RNU5A, and SNORA 73A were still ranked as the 3 most stable references, whereas $5 S$ and Met-tRNA were revealed to be the 2 least-stable genes (Figure $1 \mathrm{~b}$ ). On the other hand, a robust correlation $(\mathrm{r}=0.88, P<0.01)$ was revealed between the geNorm M-value and the NormFinder stability value by evaluating the correlation coefficient, 


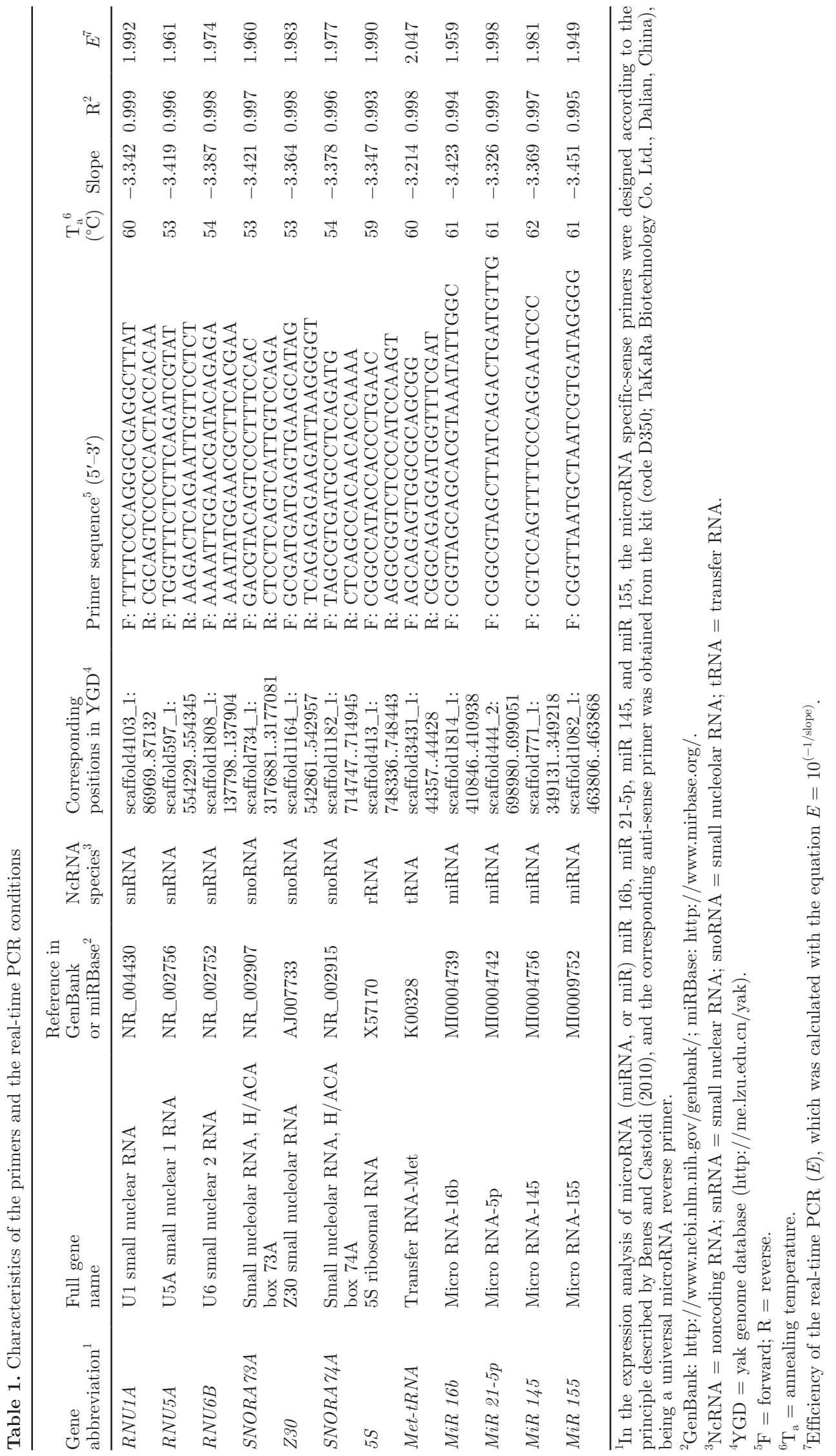


(a)

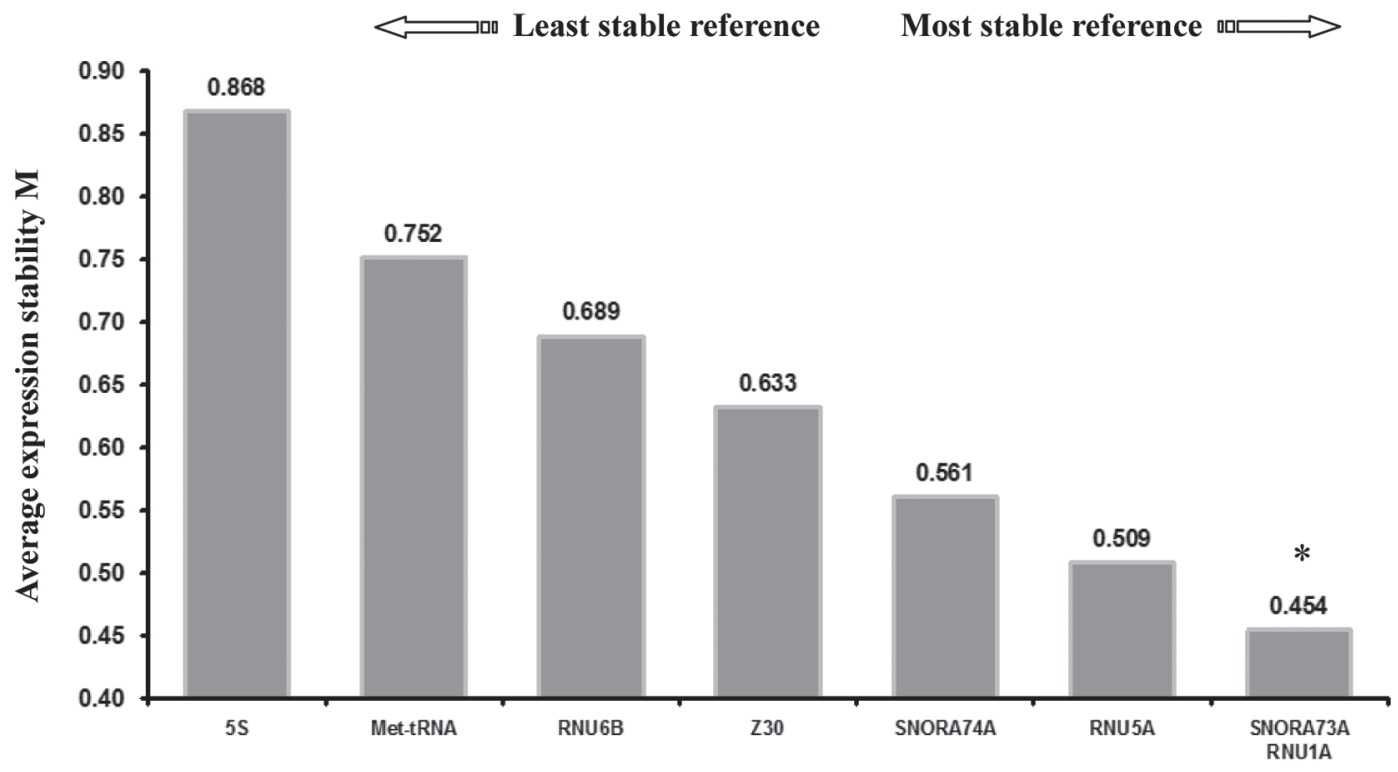

(b)

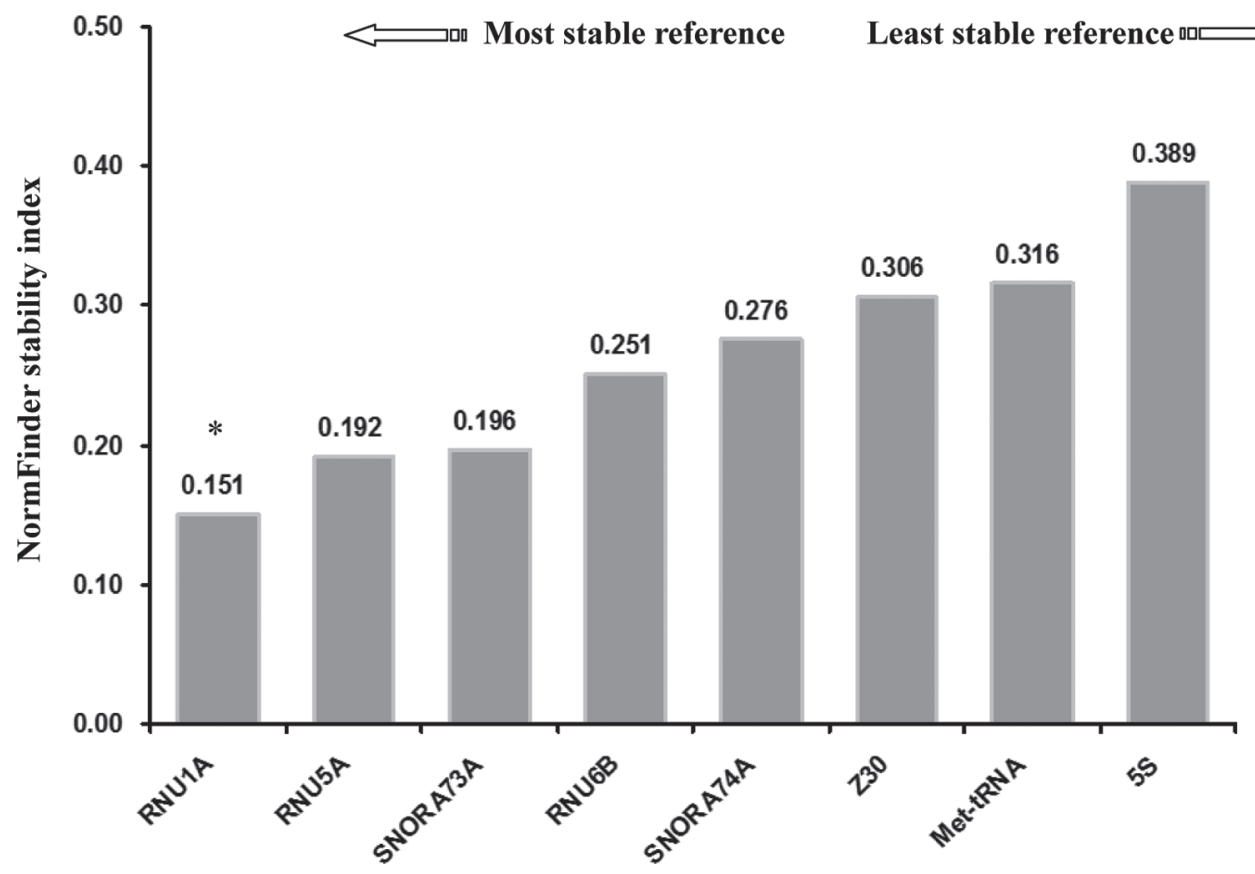

Figure 1. Stability analysis of 8 candidate noncoding small RNA based on geNorm (Vandesompele et al., 2002) and NormFinder (Andersen et al., 2004) computational program procedures. (a) Average expression stability M-values of remaining candidate references during stepwise exclusion of the least-stable reference, which was performed using geNorm procedures. The small nuclear RNA RNU1A and small nucleolar RNA SNORA73A were determined to be the most-stable reference pair, with a value of 0.454 (indicated by the asterisk). (b) Stability index values and ranking of the candidate references, carried out using the NormFinder procedure. Small nuclear RNA $R N U 1 A$ was identified as the most-stable gene, with an index value of 0.151 (indicated by the asterisk).

further confirming the reliability of our results. The geNorm analysis indicated that a minimum of 3 reference RNA were required for the normalization of microRNA expression data in milk somatic cells of lactating yaks.
Real-time PCR is a prevalent tool in the analysis of microRNA expression, and has been refined for measuring microRNA abundance (Benes and Castoldi, 2010). However, the normalization of real-time PCR data is 
(a)

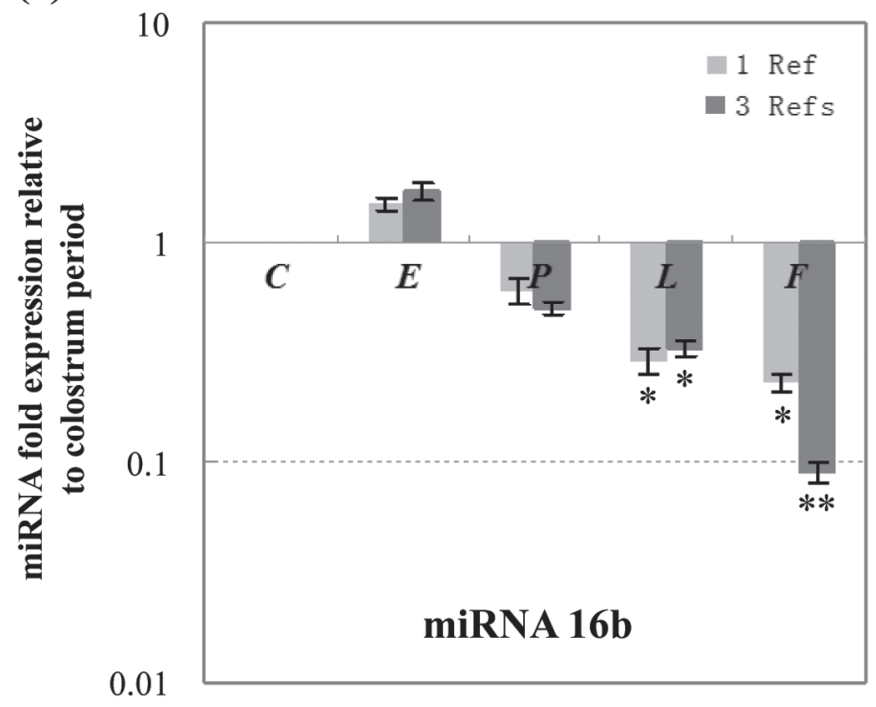

(c)

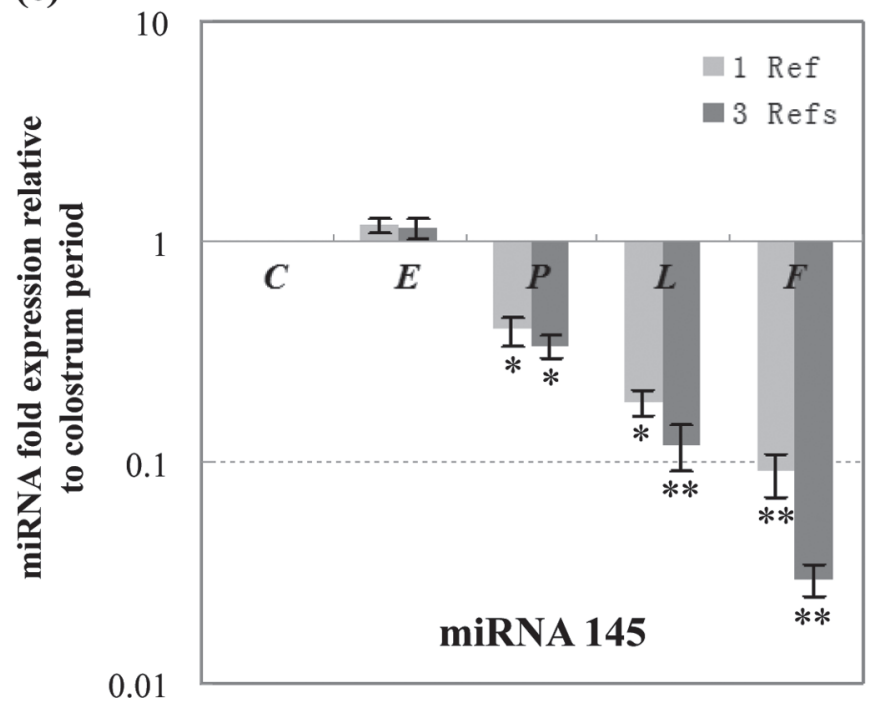

(b)

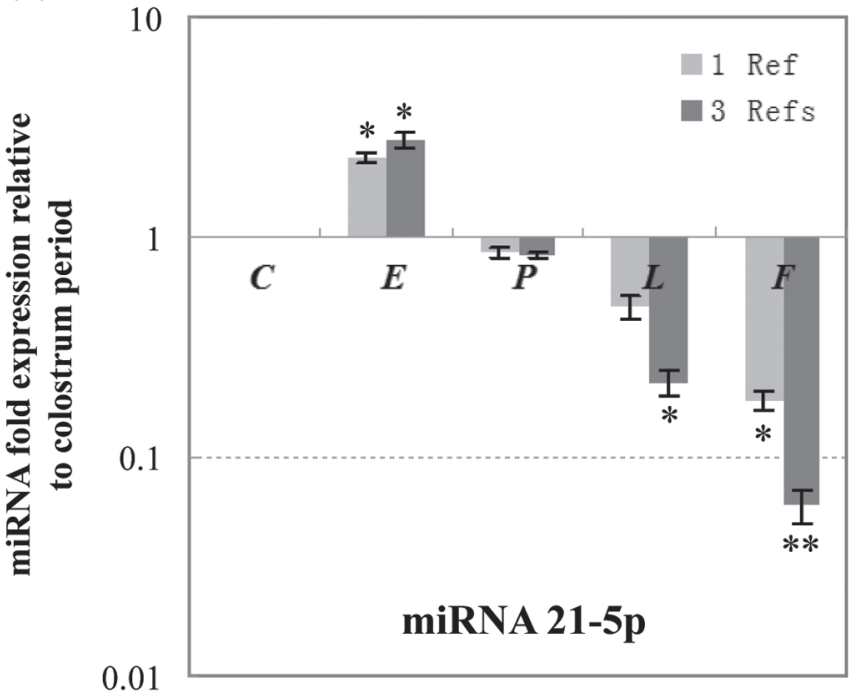

(d)

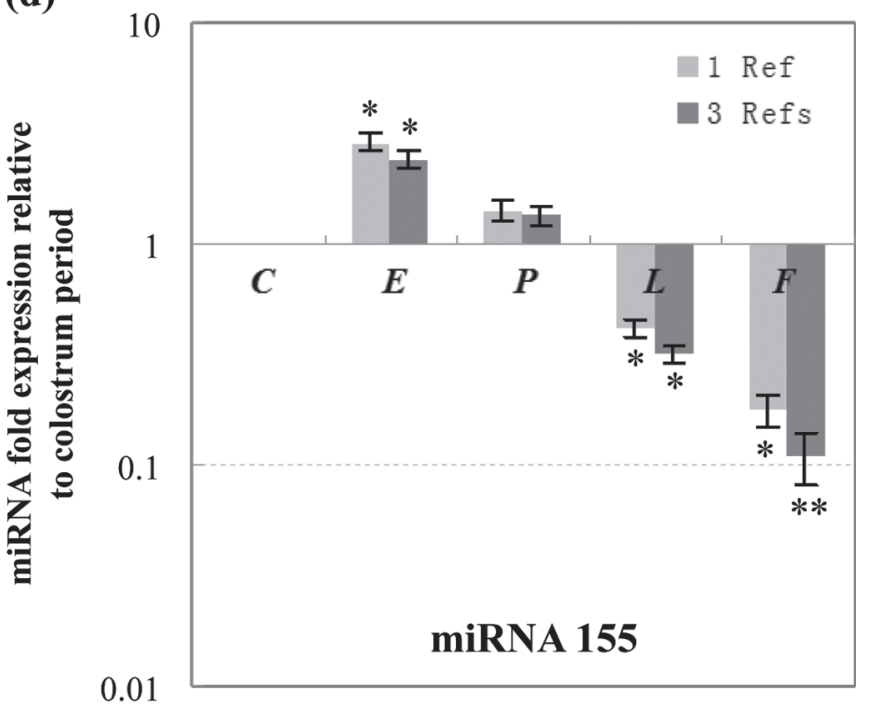

Figure 2. Relative expression of putative interest microRNA (miRNA, or miR) miR 16b (a), miR 21-5p (b), miR 145 (c), and miR 155 (d) in somatic cells of lactating yaks during early, peak, late, and final periods compared with the colostrum period. Small nuclear RNA $R N U 1 A$ alone and the combination of RNU1A, RNU5A, and small nucleolar RNA SNORA 73A were used as references. Error bars indicate the SE within a group. $C=$ colostrum period (d 3); $E=$ early period (d 28); $P=$ peak period (d 80); $L=$ late period (d 150); $F=$ final period (d 180$)$. In comparison with the colostrum period, ${ }^{*}$ indicates significant differences at $P<0.05$ and ${ }^{* *}$ indicates significant differences at $P<0.01$.

crucial to reduce possible errors. Moreover, it was demonstrated that the use of a single reference appears to be insufficient in the normalization of real-time PCR data (Vandesompele et al., 2002). In selecting suitable references for normalization of microRNA expression analysis, it is pivotal that candidate references should share similar properties with microRNA molecules, such as size. Numerous noncoding small RNA species $(<200$ bp), including rRNA, tRNA, snRNA, and snoRNA, are closer in size to microRNA and have previously been used as references in microRNA expression studies of the human breast (Davoren et al., 2008). In the present work, the 8 noncoding small RNA species were chosen mainly based on the following considerations: first, $R N U 6 B$ and $5 S$ were widely used as normalizers for microRNA expression analysis in the mammary gland of cattle and goats (Ji et al., 2012; Li et al., 2012; Wang et al., 2012), although evidence exists that these 2 RNA species (RNU6B and $5 S$ ) were not suitable for microRNA expression analysis in the pig lactating mammary gland (Gu et al., 2012). Second, RNU1A, RNU5A, and $S N O R A 73 A$ were commercially available for normaliza- 
tion of microRNA expression data from Qiagen Inc. (Mississauga, ON, Canada) and TaKaRa Biotechnology Co. Ltd. Third, Z30, SNORA74A, and Met-tRNA have been explored as candidate normalizers for studying microRNA expression in the mammary glands of humans and pigs (Davoren et al., 2008; Gu et al., 2012).

The 3 references ( $R N U 1 A, R N U 5 A$, and SNORA 73A) identified as the most stable are available commercially for normalization of microRNA expression, which will facilitate greatly the quantitative analysis of microRNA in somatic cells of lactating yaks in the future. Previously, RNU6B and $5 S$ were widely used as normalizers for microRNA expression analysis in the mammary gland of cattle (Li et al., 2012; Wang et al., 2012) and goats (Ji et al., 2012). However, our results from this work demonstrated that these 2 references (RNU6B and $5 S$ ) were not appropriate for normalization of microRNA expression data in somatic cells of lactating yaks. Particularly, it was remarkable that $5 S$ was ranked as the least-stable reference transcript in somatic cells of lactating yaks based on both geNorm and NormFinder analysis (Figure 1). Additionally, although Met-tRNA (along with $R N U 6 B$ ) was used as a reference for microRNA profiling in the various tissues of humans and mice, including the mammary gland (Liu et al., 2004), our results indicated that Met-tRNA exhibited the second-lowest stability in both geNorm and NormFinder. It was also reported that Met- $t R N A$ was unsuitable as a normalizer for microRNA expression analysis in pig mammary glands (Gu et al., 2012).

The expression abundances of $\mathrm{miR} 16 \mathrm{~b}$, miR 21-5p, miR 145, and miR 155 are presented in Figure 2. The relative expressions of the 4 microRNA were upregulated at early periods and, thereafter, were downregulated continuously until the final period, compared with the colostrum period. These results are similar to those reported recently for the bovine mammary gland (Wang et al., 2012). On the other hand, a highly similar expression pattern was indicated for each microRNA investigated between using 3 combined references and using a single one in the current study. At late periods, however, a significant difference was revealed in the relative expression of miR 21-5p compared with the colostrum period $(P<0.05)$ when using 3 references, but not when using a single reference (Figure $2 \mathrm{~b}$ ). Based on the results from this study, we recommend that the combination of RNU1A, RNU5A, and SNORA73A can be used as normalizers for microRNA quantitative analysis in future longitudinal studies on milk somatic cells of lactating yaks in relation to lactation.

\section{ACKNOWLEDGMENTS}

This work was supported financially by a grant from National R\&D Project of Transgenic Organisms of the
Ministry of Science and Technology of China (Beijing; no. 2011ZX08007-001), and the National High Technology Development Project (no. 2013AA102505). R. H. Yin was also supported financially by the Projects of Tianzhushan Yingcai of Shenyang Agricultural University (Shenyang, Liaoning, China).

\section{REFERENCES}

Andersen, C. L., J. L. Jensen, and T. F. Ørntoft. 2004. Normalization of real-time quantitative reverse transcription-PCR data: A model-based variance estimation approach to identify genes suited for normalization, applied to bladder and colon cancer data sets. Cancer Res. 64:5245-5250.

Avril-Sassen, S., L. D. Goldstein, J. Stingl, C. Blenkiron, J. Le Quesne, I. Spiteri, K. Karagavriilidou, C. J. Watson, S. Tavaré, E. A. Miska, and C. Caldas. 2009. Characterisation of microRNA expression in post-natal mouse mammary gland development. BMC Genomics 10:548.

Bai, W. L., R. J. Yang, R. H. Yin, W. Q. Jiang, G. B. Luo, R. L. Yin, S. J. Zhao, C. Li, and Z. H. Zhao. 2012. Molecular characterization and expression analysis of osteopontin cDNA from lactating mammary gland in yak (Bos grunniens). Mol. Biol. Rep. 39:3627-3635.

Benes, V., and M. Castoldi. 2010. Expression profiling of microRNA using real-time quantitative PCR, how to use it and what is available. Methods 50:244-249.

Boutinaud, M., H. Rulquin, D. H. Keisler, J. Djiane, and H. Jammes, 2002. Use of somatic cells from goat milk for dynamic studies of gene expression in the mammary gland. J. Anim. Sci. 80:12581269.

Davoren, P. A., R. E. McNeill, A. J. Lowery, M. J. Kerin, and N. Miller. 2008. Identification of suitable endogenous control genes for microRNA gene expression analysis in human breast cancer. BMC Mol. Biol. 9:76.

Du, T., and P. D. Zamore. 2005. microPrimer: The biogenesis and function of microRNA. Development 132:4645-4652.

Gu, Y. R., Y. Liang, J. J. Gong, K. Zeng, Z. Q. Li, Y. F. Lei, Z. P. He, and X. B. Lv. 2012. Suitable internal control microRNA genes for measuring miRNA abundance in pig milk during different lactation periods. Genet. Mol. Res. 11:2506-2512.

Ji, Z., G. Wang, Z. Xie, J. Wang, C. Zhang, F. Dong, and C. Chen. 2012. Identification of novel and differentially expressed microRNAs of dairy goat mammary gland tissues using Solexa sequencing and bioinformatics. PLoS ONE 7:e49463.

Li, Z., H. Liu, X. Jin, L. Lo, and J. Liu. 2012. Expression profiles of microRNAs from lactating and non-lactating bovine mammary glands and identification of miRNA related to lactation. BMC Genomics 13:731.

Liu, C.-G., G. A. Calin, B. Meloon, N. Gamliel, C. Sevignani, M. Ferracin, C. D. Dumitru, M. Shimizu, S. Zupo, M. Dono, H. Alder, F. Bullrich, M. Negrini, and C. M. Croce. 2004. An oligonucleotide microchip for genome-wide microRNA profiling in human and mouse tissues. Proc. Natl. Acad. Sci. USA 101:9740-9744.

Liu, H. N., F. Z. Ren, L. Jiang, Z. L. Ma, H. J. Qiao, S. S. Zeng, B Z. Gan, and H. Y. Guo. 2011. Fatty acid profile of yak milk from the Qinghai-Tibetan Plateau in different seasons and for different parities. J. Dairy Sci. 94:1724-1731.

Pfaffl, M. W. 2001. A new mathematical model for relative quantification in real-time RT-PCR. Nucleic Acids Res. 29:e45.

Vandesompele, J., K. De Preter, F. Pattyn, B. Poppe, N. Van Roy, A. De Paepe, and F. Speleman. 2002. Accurate normalization of real-time quantitative RT-PCR data by geometric averaging of multiple internal control genes. Genome Biol. 3:research0034.1research0034.11.

Wang, M., S. Moisá, M. J. Khan, J. Wang, D. Bu, and J. J. Loor. 2012. MicroRNA expression patterns in the bovine mammary gland are affected by stage of lactation. J. Dairy Sci. 95:6529-6535. 